\title{
On feasibility of friction stir processing of cylindrical hole
}

N J Panaskar, A Sharma

Indian Institute of Technology Hyderabad, India

\begin{abstract}
A novel application of the Friction stir processing (FSP) in surface modification of walls of the cylindrical hole is presented. Predrilled holes in commercially available aluminum plate were friction stirred using a specially fabricated tapered tool that rotates and simultaneously traverses through the hole. The tapered tool expands the hole to the desired diameter and also stirs the internal wall of the hole. In order to understand the role of frictional heating and stirring in the FSP of holes three mediums, namely, dry (without cooling and lubricating), metal working fluid (cooling and lubricating) and $\mathrm{Al}_{2} \mathrm{O}_{3}$ nanoparticles (lubricating and frictional heating) were used. The surface roughness and hardness of the wall of the holes were measured. Significant hardness improvement with reasonable surface finish is obtained. Moreover the FSPed holes showed improvement in the fatigue life of the holes. The results are promising enough however many more investigation need to be carried out to fully understand the physics of the process and subsequently apply the same at the shop-floor.
\end{abstract}

Keywords: Friction stir processing, burnishing, hardness, surface finish, fatigue life.

\section{Introduction}

Fastener holes are used widely in various applications such as aerospace and automobile. The components used with such holes may be retired from service due to wear, corrosion, and cracks. The component life can be improved by enhancing the overall mechanical properties of the hole surface as well as those in the region which is in the vicinity of the hole. Mechanical properties of the holes can be enhanced by improving hardness, surface finish and small amount of residual stress by using combined Friction Stir Processing (FSP) and burnishing process. FSP is a modification of Friction Stir Welding (FSW) process which was invented by The Welding Institute (TWI) in 1991. FSW uses a rotating pin tool which is inserted into the joint until the tool shoulder touches the workpiece surface and this tool traverses along the joint line. Heat generated by frictional heating and the plastic deformation softens the material around the vicinity of the tool. The stirring effect along the joint interface produced by the tool results in a solid-state weld. Other variants of FSW process include Hybrid Friction Stir welding, Friction stir spot welding, Self-reacting FSW and Friction Stir Processing/ Surfacing.

FSP is well known as a surface modification technique based on $\mathrm{FSW}^{1)-5}$. It has been primarily developed for eliminating several defects in cast products or improving mechanical properties of light metals and their alloys by grain refinement related to the HallPetch relation. Recently, the FSP has been studied as a process for producing surface nano-composite on flat plates. The FSP, being a low temperature process operation should be an appropriate process to disperse the nano composite particles into the metals. Aluminum matrix composites (AMC) possess the stiffness, hardness and strength of hard ceramic reinforcements as well as the toughness of the alloy matrix ${ }^{6)-8)}$. The major advantages of AMCs are as follows: better strength and hardness, increased stiffness, reduced density, good corrosion resistance, improved Young's modulus, better formability at higher temperatures, improved superplasticity, increased wear resistance and damping capabilities ${ }^{6)-}$ 13). The following section gives background of the present investigation.

\section{Background}

FSP studies done so far mainly deal with aluminum and its alloys. The tool geometry, the base material, nano-sized powder and the number of passes performed were the important parameters in these studies. Lee et al. ${ }^{14)}$ used $\mathrm{SiO}_{2}$ nano-sized powder in a groove made in AZ61 Mg Alloy plate and performed four FSP passes. They observed that the hardness of the composites was almost twice that of the base metal and it also exhibited high strength and strain rate superplasticity respectively. Shafiei et al. ${ }^{15)-16)}$ observed greater hardness and superior wear resistance during fabrication of $\mathrm{Al} / \mathrm{Al}_{2} \mathrm{O}_{3}$ nanocomposite surface layer on an $\mathrm{Al}$ alloy substrate. Aluminum plates with pre-placed nano-sized $\mathrm{Al}_{2} \mathrm{O}_{3}$ powder which was filled in a groove were subjected to various numbers of passes from one to four. They observed that the mean hardness value of the surface nano-composite layer improved by almost three times as compared to that of the as-received $\mathrm{Al}$ alloy substrate.

Zahmatkesh et al. ${ }^{17)}$ used air plasma to spray Al$10 \% \mathrm{Al}_{2} \mathrm{O}_{3}$ powder to produce $\mathrm{Al}-10 \% \mathrm{Al}_{2} \mathrm{O}_{3}$ coating on Al2024 substrate. The coated material was then subjected to FSP. They found significant improvement in hardness and wear resistance of surface 
nanocomposite respectively. Faraji et al ${ }^{18)}$ filled $\mathrm{Al}_{2} \mathrm{O}_{3}$ nano-sized powder into a groove of AZ91 plate and then FSP was carried out. They observed improvement in hardness and wear resistance. Ke et al. ${ }^{19)}$ studied FSP on Al alloy with Ni powder. The nickel powder was filled into two rank holes on two matrix plate before FSP. They reported significant improvement in hardness and tensile strength. Sun et al. ${ }^{20)}$ used two pure $\mathrm{Cu}$ plates clamped together on the back plate with a $1 \mathrm{~mm}$ wide gap between the two adjoining plates in which $\mathrm{SiC}$ particles were inserted and pressed tightly. They observed significant improvement in hardness after two passes of FSP. Shamsipur et al. ${ }^{21)}$ performed FSP on commercially pure titanium substrate using nano-sized $\beta$-SiC powder inserted in a groove made along the middle length of each work piece. They observed an improvement in hardness of the $\mathrm{Ti} / \mathrm{SiC}$ nano-composite surface layer by about 3.3 times greater as compared to the asreceived Ti substrate.

All the above-mentioned studies which have been discussed involve FSP performed on flat plates. However, there is no literature available as yet on FSP performed on cylindrical surfaces. The present study is supposed to be the first one which investigates the possibility of enhancing the surface properties of a cylindrical cavity. The specially designed FSP tool is given a feed in the vertical direction so that it moves downward inside the cylindrical cavity, thereby applying pressure and frictional force on the internal surface of the cavity, instead of moving transversely as in the case of flat plate FSP. In flat plate FSP, the nano-sized powder is placed in the groove formed between the plates along the FSP direction. Alternatively, the powder is mixed with volatile solvents like methanol and applied to the surface of plates and subsequently performing FSP. However, in the present process, the nano-sized powder is filled using a funnel which replenishes the powder which gets pressed due to the pressure of the tool in the downward direction and on the internal side of the cavity. The process can also be carried out using metal working fluid as a cooling and lubricating medium as well as in dry condition. The FSP operation is followed by the burnishing operation. Moreover, the drilling, FSP and burnishing operations should be done subsequently without altering the position of the work piece to get the best results. The following section gives the details on material and experimental method.

\section{Material and experimental method}

The work specimens employed for experiments were made of commercially available pure Aluminium of 6 $\mathrm{mm}$ thickness. Work samples had width $50 \mathrm{~mm}$ and length $100 \mathrm{~mm}$. The chemical composition for the material used is shown in Table 1. Commercially available nano-sized alumina powder $($ size $<50 \mathrm{~nm})$ was used. The basic design of the tool used for FSP was in form of a tapered mandrel. The bottom diameter of the tool was $9.5 \mathrm{~mm}$ while the upper diameter was $10 \mathrm{~mm}$. There was a tapered knurled portion for a length $15 \mathrm{~mm}$ until the final diameter is $10 \mathrm{~mm}$ which extends for the rest of the tool length. The work sample plate at the top was used as a dummy to aid the FSP operation. A funnel shaped structure was fixed onto the top dummy plate to allow the $\mathrm{Al}_{2} \mathrm{O}_{3}$ nano-sized powder to enter the open space between the tool and the cavity during the FSP operation.

Table 1. Chemical composition of material used

\begin{tabular}{lcccccc}
\hline Material & $\mathrm{Al}$ & $\mathrm{Cu}$ & $\mathrm{Fe}$ & $\mathrm{Mn}$ & $\mathrm{Si}$ & $\mathrm{Zn}$ \\
\hline $\begin{array}{l}\text { Commercial } \\
\begin{array}{l}\text { Pure } \\
\text { Aluminium }\end{array}\end{array}$ & 98.95 & 0.005 & 0.457 & 0.014 & 0.564 & 0.006 \\
\hline
\end{tabular}

The FSP was done in three different conditions: with metal working fluid (hereafter referred to as "wet condition'), without metal working fluid (hereafter referred to as 'dry condition') and dry with nano-sized particles (hereafter referred to as 'nano condition'). Conventional water soluble coolant was used for this purpose. After the drilling operation, the over-sized rotating tool was inserted through the hole at a rotating speed of $60 \mathrm{rpm}$ and a feed of $8 \mathrm{~mm} / \mathrm{min}$ and then retracted back with the same parameters. The FSP operation was followed by the burnishing process. The process flow is shown in figure 1 . The same procedure was used for experiments performed under dry and wet conditions.

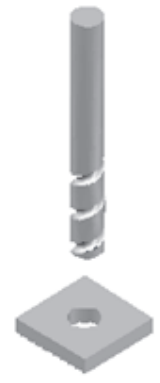

(a)

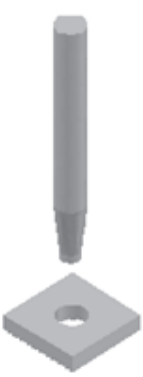

(b)

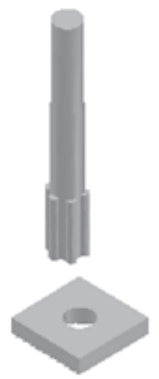

(c)
Fig. 1. Process flow - (a) Drilling, (b) FSP, (c) Burnishing

Two types of experiments were done: One involving FSP and another one involving combined FSP with burnishing. A roller burnishing tool was used for the burnishing operation. The rotational speed used for burnishing was $2000 \mathrm{rpm}$ and the feed rate was 1000 $\mathrm{mm} / \mathrm{min}$.

Surface roughness was evaluated using a Mahr surface roughness tester (Marsurf XR 20). The FSP operated samples were cut vertically into two equal halves along the centre of the cavity to measure the hardness. This was done using wire-Electrical Discharge machine so that the hardness is minimally altered by the cutting process. The hardness of surface composite layers across the friction zone was measured using a Vickers hardness tester with a load of $100 \mathrm{kgf}$. Each hardness value is the average of three measurements. Fatigue specimens of aluminium samples were produced as per ASTM E 466-07. The fatigue life 
assessment was carried out using a $100 \mathrm{kN}$ capacity MTS UTM machine. The maximum stress value is kept at $105.6 \mathrm{MPa}$ (nearly 0.9 times the yield strength) for fatigue test. The fatigue tests were carried out using constant amplitude, sinusoidal cyclic loads with a load ratio of $\mathrm{R}=0.1$ (Pmin/Pmax). A load frequency of $25 \mathrm{~Hz}$ was applied on the sample.

\section{Results and Discussion}

The surface roughness showing $\mathrm{Ra}$ values are shown in figure 2. The surface roughness of the samples operated under dry and wet conditions is lower compared to that of samples which are plain drilled without using coolant. The nanoparticle operated samples has surface roughness nearly same as that of the samples which are plain drilled without using coolant. After the burnishing process is employed, the surface roughness of the nanoparticle operated sample is reduced significantly to less than half the roughness of samples which are plain drilled without using coolant. The samples plain drilled with coolant have the least surface roughness.

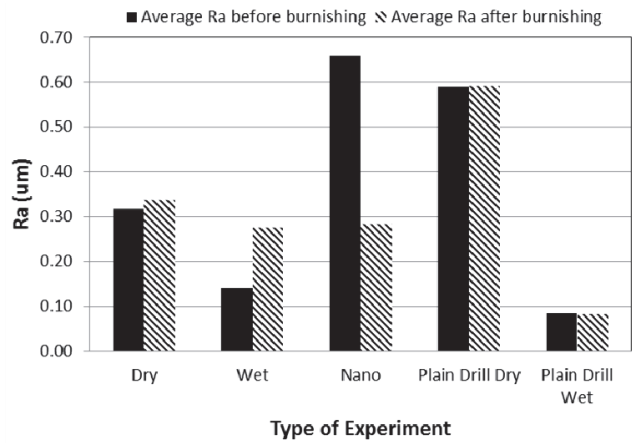

Fig. 2. Surface roughness (Ra)

The hardness values are shown in figure 3. The combined FSP with burnishing process results in an increase in hardness. The increase in hardness over the samples drilled with coolant is found to be highest, about $67.6 \%$, in case of FSP with nanoparticles. This increase in hardness is mainly due to the the embedding of nanoparticles over the hole surface. It is important to note that there is not much change in hardness due to burnishing, however, the surface finish improved significantly as seen earlier.

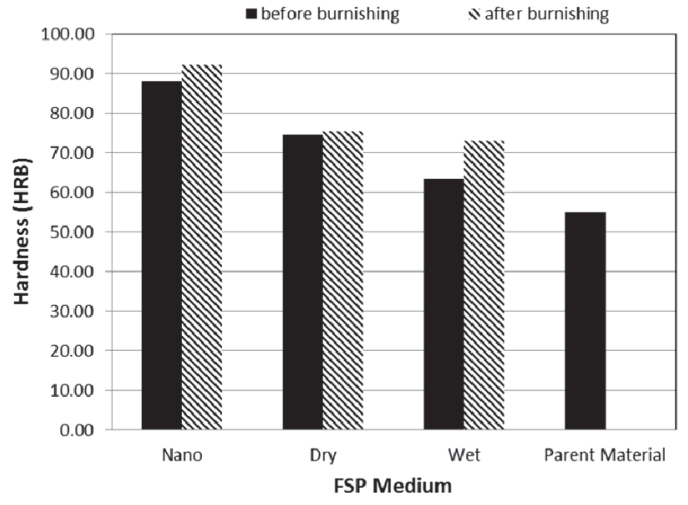

Fig. 3. Hardness measurement
Slight amount of grain refinement also occurs due to the tool stirring action which is evident from the hardness improvement with dry and wet conditions. There was approximately $36.9 \%$ increase in hardness in case of FSP under dry conditions. There was no significant increase in hardness under wet condition before burnishing. The reason for this could be the loss of hardness due to the elastic spring back of the material around the vicinity of the hole after the FSP operation.

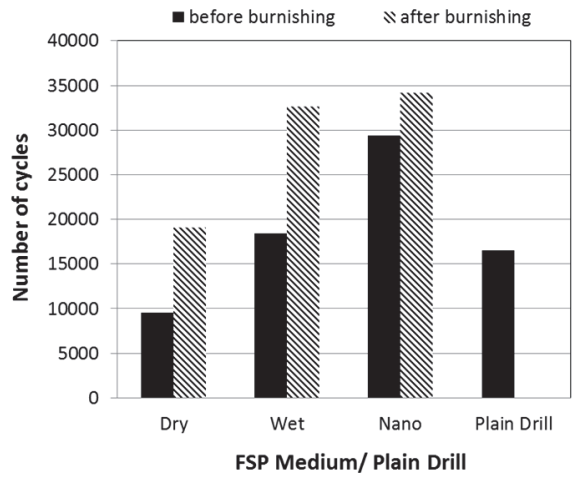

Fig. 4. Fatigue Life

The number of cycles sustained under the fatigue loading before and after burnishing is presented in figure 4 . The dry FSP sample without burnishing fails very early, even earlier than the plain drilled hole. The wet and dry samples have almost same hardness, as shown in the figure 3; however, the surface of dry samples is comparatively rough that seems to be the probable reason for early failure. On the other hand, the surface is comparatively rough in case the nano FSPed samples, however they sustain maximum number of cycles before failure. The embedding of nanoparticles seems to be responsible for this observation. The nanoparticles generate friction resulting in softening of base material, as seen in the figure 5 showing SEM images of FSPed surfaces. This allows embedding of nanoparticles in the microcracks. This restricts closing of the crack in the compression half cycle; thus, the fatigue life of the component improves.

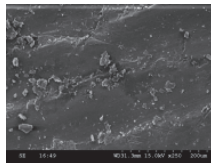

Dry

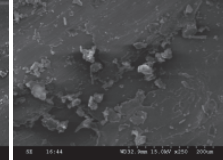

Wet

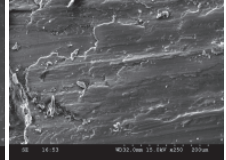

Nano
Fig. 5. SEM images of FSPed surfaces

\section{Conclusion}

A novel application of friction stir processing is presented. The surface properties of the wall of cylindrical hole can be altered by friction stir processing using a specially designed tool that rotates while traversing through a cylindrical hole. The developed approach can further be extended to aerospace materials. The variation due to process parameters such as feed rate and rpm rotational speed 
needs to be studied. The following are the main conclusions of the present work:

1. The performance of the FSP of cylindrical hole is highly influenced by the medium between the tool and the workpiece.

2. The FSP without any external medium results in reduction in fatigue life because of the microcracks developed on the surface in absence of cooling and lubricating medium.

3. The FSP with wet medium marginally improves the hardness and the fatigue life as well. However, burnishing after the FSP improves the fatigue life.

4. There was significant improvement in hardness and fatigue life in case of FSP with nanoparticles even without burnishing.

\section{Acknowledgement}

The authors acknowledge funding from IIT Hyderabad provided under the seed-grant project - Friction stir surfacing of metallic and non-metallic surfaces.

\section{References}

[1] H. Fujii, Y. G. Kim, T. Tsumura, T. Komazaki, and K. Nakata, Estimation of material flow in stir zone during friction stir welding by distribution measurement of $\mathrm{Si}$ particles, Materials transactions, 47, 1 (2006), 224-232.

[2] H. J. Liu, H. Fujii, and K. Nogi, Microstructure and mechanical properties of friction stir welded joints of AC4A cast aluminium alloy, Materials science and Technology, 20, 3 (2004), 399-402.

[3] K. Oh-Ishi and T. McNelley, Microstructural modification of as-cast NiAl bronze by friction stir processing, Metallurgical and Materials Transactions A, 35, 9 (2004), 2951-2961.

[4] D. C. Hofmann and K. S. Vecchio, Submerged friction stir processing (SFSP): An improved method for creating ultra-fine-grained bulk materials, Materials Science and Engineering: A, 402, 1-2 (2005), 234-241.

[5] J.Q. Su, T. W. Nelson, and C. J. Sterling, Friction stir processing of large-area bulk UFG aluminum alloys, Scripta Materialia, 52, 2 (2005), 135-140.

[6] J. Torralba, C. da Costa, and F. Velasco, P/M aluminum matrix composites: an overview, Journal of Materials Processing Technology, 133, 1-2 (2003), 203-206.

[7] G. Abouelmagd, Hot deformation and wear resistance of $\mathrm{P} / \mathrm{M}$ aluminium metal matrix composites, Journal of Materials Processing Technology, 155-156, (2004), 1395-1401.

[8] S. Das, S. Das, and K. Das, Abrasive wear of zircon sand and alumina reinforced $\mathrm{Al}-$ $4.5 \mathrm{wt} \% \mathrm{Cu}$ alloy matrix composites - A comparative study, Composites Science and Technology, 67, 3-4 (2007), 746-751.
[9] S. Candan and E. Bilgic, Corrosion behavior of Al-60 vol.\% SiCp composites in $\mathrm{NaCl}$ solution, Materials Letters, 58, 22-23 (2004), 2787-2790.

[10] S. Chaudhury, A. Singh, C. Sivaramakrishnan, and S. Panigrahi, Preparation and thermomechanical properties of stir cast $\mathrm{Al}-2 \mathrm{Mg}$ $11 \mathrm{TiO}_{2}$ (rutile) composite, Bulletin of Materials Science, 27, 6 (2004), 517-521.

[11] S. Sawla and S. Das, Combined effect of reinforcement and heat treatment on the two body abrasive wear of aluminum alloy and aluminum particle composites, Wear, 257, 5-6 (2004), 555-561.

[12] A. M. Hassan, G. M. Tashtoush, and J. A. AlKhalil, Effect of Graphite and/or Silicon Carbide Particles Addition on the Hardness and Surface Roughness of Al-4 wt\% Mg Alloy, Journal of Composite Materials, 41, 4 (2007), 453-465.

[13] R. Bauri, D. Yadav, and G. Suhas, Effect of friction stir processing (FSP) on microstructure and properties of $\mathrm{Al}-\mathrm{TiC}$ in situ composite, Materials Science and Engineering: A, 528, 1314 (2011), 4732-4739.

[14] C. J. Lee, J. C. Huang, and P. J. Hsieh, Mg based nano-composites fabricated by friction stir processing, Scripta Materialia, 54, 7 (2006), $1415-1420$.

[15] A. Shafiei-Zarghani, S. F. Kashani-Bozorg, and A. Zarei-Hanzaki, Microstructures and mechanical properties of $\mathrm{Al} / \mathrm{Al} 2 \mathrm{O} 3$ surface nanocomposite layer produced by friction stir processing, Materials Science and Engineering: A, 500, 1-2 (2009), 84-91.

[16] A. Shafiei-Zarghani, S. F. Kashani-Bozorg, and A. Z.- Hanzaki, Wear assessment of Al/A12O3 nano-composite surface layer produced using friction stir processing, Wear, 270, 5-6, (2011), 403-412.

[17] B. Zahmatkesh and M. H. Enayati, A novel approach for development of surface nanocomposite by friction stir processing, Materials Science and Engineering: A, 527, 2425 (2010), 6734-6740.

[18] G. Faraji and P. Asadi, Characterization of AZ91/alumina nanocomposite produced by FSP, Materials Science and Engineering: A, 528, 6 (2011), 2431-2440.

[19] L. Ke, C. Huang, L. Xing, and K. Huang, Al-Ni intermetallic composites produced in situ by friction stir processing, Journal of Alloys and Compounds, 503, 2 (Aug. 2010), 494-499.

[20] Y. F. Sun and H. Fujii, The effect of SiC particles on the microstructure and mechanical properties of friction stir welded pure copper joints, Materials Science and Engineering: A, 528, 16-17 (2011), 5470-5475.

[21] A. Shamsipur, S. F. Kashani-Bozorg, and A. Zarei-Hanzaki, The effects of friction-stir process parameters on the fabrication of $\mathrm{Ti} / \mathrm{SiC}$ nano-composite surface layer, Surface and Coatings Technology, 206, 6 (2011), 1372-1381. 